\title{
Théodore Agrippa d'Aubigné charmé par les voix du mythos
}

\author{
CAROLE DUCHESNE
}

\section{P}

armi les grands personnages de la scène littéraire française de la seconde partie du seizième siècle, Agrippa d'Aubigné fut, chez les Réformés, la figure la plus marquante. Guerrier et poète, il participa, à ces deux titres, aux confrontations de son temps. D'ailleurs, bien que publiés qu'en 1616, Les Tragiques furent écrits dans la fureur des combats alors que l'auteur, immobilisé par une blessure reçue aux combats de Casteljaloux (1577), indigné, tourmenté, en colère, troquait l'épée contre la plume et rédigeait ce qu'il concevait comme le cri de ralliement des vrais croyants. Les Tragiques se veulent un grand poème épique destiné à la défense et à l'illustration de l'Église réformée. ${ }^{1}$ Porté par sa foi et son art, Agrippa d'Aubigné entreprend sciemment de démontrer la prééminence de l'Église réformée. D'ailleurs, il nous explique longuement, dans son introduction, le but de son entreprise et les moyens qu'il entend utiliser.

La matiere de l'oeuvre a pour sept livres sept tiltres separez, qui toutefois ont quelque convenance, comme ses effects aux causes. ${ }^{2}$

Il construit cette défense selon les règles de l'art juridique - rappel des crimes commis contre ses co-religionnaires dans les Misères, identification des coupables et accusation dans Les Princes et dans La chambre dorée, appel des témoins dans les Feux et les Fers, rappel des faits et plaidoirie dans Vengeance et enfin le Jugement. Toute l'articulation de ce discours vise à convaincre le lecteur qu'il vaut mieux souffrir ici-bas, comme c'est le cas des Réformés, et être parmi les bienheureux éternellement.

Il ne faut esgaller à l'éternelle peine

Et aux soupirs sans fin un poinct de courte haleine. ${ }^{3}$

La structure des Tragiques ainsi que le récit du destin des vrais croyants, victimes des puissants de la terre mais élus de Dieu, respectant les lois du logos: elle vise à convaincre. Et pourtant, malgré cette volonté manifeste 
d'Agrippa d'Aubigné de s'en tenir à une parole fondée sur des présuppositions dont, au seizième siècle, la justesse n'était point contestée, à savoir que le schéma divin de la vie est la rédemption du pécheur et son retour au royaume céleste d'où Adam et Eve ont été chassés, il ne semble pas qu'il ait pu échapper aux rythmes primes de l'immense, du compliqué palimpseste de la mémoire primordiale.

La parole n'est point univoque et c'est pourquoi la langue grecque ancienne dispose pour elle de toute une série de désignations: phônêma est la parole en tant qu'elle est ébruitement vocal; logos est la parole en tant qu'elle est pesée, sensée; mythos est la parole en tant que témoignage immédiat de ce qui fut, est et sera, révélation de l'être au sens véritable qui ne distingue pas entre la parole et l'être. Mu par sa foi et armé de son art, comment Agrippa d'Aubigné aurait-il pu rester sourd aux voix du mythos; comment aurait-il pu résister aux charmes des muses? La religion et l'art du poète furent, selon nous, les brèches par lesquelles le mythos s'infiltra jusqu'à coloniser Les Tragiques, cette oeuvre qui se voulait un plaidoyer soumis aux seules lois du logos.

\section{Toute religion est mythique}

Le récit qui nous est fait dans Les Tragiques se plie aux lois de la causalité: souillé par la faute d'Adam et Eve, l'homme appelle, du fond de sa misère, la rédemption. Le vrai croyant aspire à la perfection du temps d'avant la chute, du temps d'avant le péché, du temps des origines. ${ }^{4}$ Et c'est sous l'oeil du Dieu unique qu'il entreprend le difficile voyage qui le ramènera à la maison du Père, au royaume perdu. Or, la croyance en la perfection des origines et, par conséquent, en la nécessité d'y retourner échappe totalement aux lois du logos selon lesquelles le processus, le devenir, l'évolution corrigent, peu à peu, la pénible pauvreté des commencements. Les lois du logos sont celles du progrès, tandis que la croyance en la béatitude des commencements participe de la révélation. Dieu peut seulement être éprouvé "en l'obscur des esprits"5 ou non.

Sa manifestation au début des temps avait nom de mythe. Tous les mythes sans exception gravitent autour d'un vide, d'un inconnu, innommé, innommable, dieu. Sans théophanie, il n'y a pas de mythe. Mythe est le nom de tout ce qui existe et subsiste ayant la parole pour cause. Mythos est la parole vraie, non au sens de ce qui a force de preuve, mais au sens du donné factuel, de ce qui s'est révélé, de ce qui est vénéré. C'est ce par quoi cette parole se distingue de toute autre énonciation. ${ }^{6}$ Le mythe des origines ne représente pas le divin de façon imagée, ne symbolise pas le divin, il est l'être du divin manifesté. 
L'idée de la perfection des commencements est l'expression d'une experience religieuse intime et profonde, nourrie par le souvenir imaginaire d'un paradis perdu, d'une béatitude qui précédait l'actuelle condition humaine. Il faut récupérer le temps originel, fort, sacré; il faut donc retourner à la maison du Père puisque "C'est de tout l'avenir le registre". ${ }^{7}$

Et c'est là, au coeur même du mythe, au temps sacré de l'origine, manifestation primordiale de l'être divin, qu'Agrippa d'Aubigné, prophète, conduit ses ouailles. Guidé par sa foi de Réformé, Agrippa d'Aubigné peut, grâce aux lumières de l'Écriture, nommer le chaos qui l'entoure - il a nom d'antéchrist. Le règne de l'antéchrist n'équivaut-il pas à un retour au chaos? Ce monde-ci - le monde l'histoire - est injuste, abominable, démoniaque; heureusement il craque de toutes parts; très prochainement, ce vieux monde sera anéanti, les forces des ténèbres seront définitivement vaincues, les bons triompheront, le paradis sera retrouvé. Puisqu'il y a eu commencement, il doit y avoir fin. La fin est partie intrinsèque du commencement et vice versa. Pour qu'un commencement absolu soit, il faut que la fin du monde soit radicale. L'eschatologie n'est que la préfiguration d'une cosmogonie de l'avenir, le triomphe d'une histoire sainte. Il ne s'agit pas de regénérer ce qui est dégénéré, mais d'anéantir le vieux monde afin de pouvoir le recréer in toto. D'où la nécessité de mourir à ce monde dénaturé pour renaître à la pureté de la nature originelle. Pour Agrippa d'Aubigné, comme pour tous les tenants des religions judéochrétiennes, il n'y aura qu'une seule fin du monde tout comme il n'y a eu qu'une seule cosmogonie. ${ }^{8}$ Aussi, est-ce de tout son coeur qu'il appelle la fin de ce monde:

[...] "Vien Seigneur et te haste

Car l'homme de peché ton Eglise degaste."

"Vien", dit l'esprit, "acours pour defendre le tien."

"Vien", dit l'espouse, et nous avec l'espouse: "Vien!"

Et ce paradis, à jamais, sera. ${ }^{10}$

A la fin des temps, seuls les élus vivront dans une éternelle béatitude. Aux prises avec les tribulations et les tentations de ce monde-ci, eux seuls, parce que prédestinés, auront su rester fidèles au royaume céleste. Le salut leur sera consenti au nom même de leur fidélité à une histoire sainte, à une histoire révélée.

"Je te permets, Satan", dit l'Eternel alors,

"D'estreindre par le fer la pluspart de leurs corps;" 11

Il lui rappelle toutefois: 
Ton filet n'enclorra que les abandonnés

Qui furent nés pour toi $[\ldots]^{12}$

Ce royaume leur fut révélé et ils reçurent cette révélation avec gratitude et docilité. La connaissance de ce royaume céleste et de ses lois n'est pas le résultat d'une réflexion, étant donné que la vérité qu'il contient et qu'il est n'a pas été conclue au terme d'une démarche de la pensée. Loin d'être saisi et appréhendé par l'homme, c'est le mythe, figure de Dieu, qui saisit et appréhende, voire ébranle, l'homme lui-même, c'est la grâce. La vérité ne devient donc pas manifeste à l'homme à la suite de sa propre investigation; la vérité se révèle elle-même et Agrippa d'Aubigné, porte-étendard de la foi réformée, la proclame.

\section{Tout poète authentique n'est que l'interprète des Muses}

C'est sous la protection de la muse qu'Agrippa d'Aubigné, poète féru de culture grecque, inscrit son oeuvre, plus précisément sous celle de Melpomène, muse de la tragédie. Il faut se rappeler que les Olympiennes, les muses, sont immédiatement solidaires de Zeus, créateur, ordonnateur du monde. Leur naissance telle que racontée dans l'Hymne à Zeus de Pindare, suit l'agencement du monde et du royaume des dieux ainsi que les noces de Zeus et Themis dont-naîtront les Heures et les Moires. Lorsque Zeus demande aux dieux s'il manque encore quelque chose au cosmos, ils réclamèrent la création d'un dieu pour célébrer la constitution et la profondeur divine du monde. Cette dernière oeuvre parachève l'oeuvre de la création et porte à son achèvement l'être des choses. Que la vérité doive se révéler, les Grecs l'ont reconnu et l'ont vu comme un avènement à l'intérieur du divin. ${ }^{13}$ Ce n'est donc pas l'homme lui-même qui trouve les mots de son crû pour dire l'être et ce qu'il a de divin. La muse chante, l'homme se contente de chanter d'après elle. ${ }^{14}$ L'apparition de la muse signifie la naissance de la parole au sein de laquelle l'être des choses, le mythe, devient manifeste comme figure. C'est là une réponse grecque à la question de savoir comment l'être (le mythe divin) se révèle plastiquement et se destine à l'homme.

Agrippa d'Aubigné assume le rôle de l'aède, serviteur consentant du souffle de l'être révélé, de Melpomène, muse de la tragédie. Or, c'est par la tragédie que les Grecs répondirent au deuil d'un monde d'où "dieu se retire," lui dont la figure avait resplendi dans le mythe. La tragédie, dans l'échelle du surhumain, succède au mythe quand ce dernier se vide de sa substance et devient fable. Et c'est bien d'un monde d'où Dieu s'est retiré qu'Agrippa d'Aubigné veut nous entretenir. 
Dieu voulut en voir plus, mais de regret et d'ire

Tout son sang escuma: il fuit, il se retire,

Met ses mains au devant de ses yeux en courroux.

Le Tout-Puissant ne peut resider entre nous. ${ }^{15}$

Toute création authentique présuppose une illumination ayant sa source dans l'être des choses, une révélation du monde qui appelle comme réponse la construction d'une oeuvre. ${ }^{16}$ Le poète véritable ne choisit pas d'écrire. C'est sous la dictée de Dieu qu'Agrippa d'Aubigné écrit Les Tragiques.

Je t'ay guidé au cours du celeste voyage,

Escrits fidelement: que jamais autre ouvrage,

[...], ne te semble plaisant

[...]

Ne chante que de Dieu $[\ldots]^{17}$

L'être divin trouve une configuration dans la parole. Les images et les figures sont pour ainsi dire des incantations magiques adressées au monde de l'être, à un monde qui doit éclore, rejeter le voile épais qu'ont tissé concepts de l'entendement et raisonnements utilitaires, et surgir en sa forme originelle qui ne témoigne que pour elle-même et nous ébranle soudain jusqu'au tréfonds de notre être. Les prétendues images n'ont sur nous pouvoir qu'à la mesure de leur teneur en réalité. Et nous voilà du coup dans le domaine du mythos, du mythe tel qu'encore aujourd'hui il nous parle. Rappelons-nous que Les Tragiques furent, de l'aveu répété de l'auteur, écrits alors qu'il était dans un état de grande vulnérabilité, dans un songe, sous l'effet d'une vision.

La sensibilité d'Agrippa d'Aubigné, exacerbée par les maux qui assaillent "France" et l'Église réformée, fait de lui un chantre particulièrement perméable aux rythmes primes de l'être réfracté au miroir de la ressemblance, alors valeur structurante. ${ }^{18}$ C'est elle, en effet, qui organise le jeu des symboles et permet la connaissance des choses visibles et invisibles tout en guidant l'art de les représenter.

Agrippa d'Aubigné vit au centre d'un monde où la similitude se superpose à l'herméneutique - l'ensemble des connaissances et des techniques qui permettent de faire parler des signes et de découvrir leur sens - et à la sémiologie - l'ensemble des connaissances et des techniques qui permettent de distinguer où sont les signes, de définir ce qui les constitue comme signes, de connaître leurs liens et les lois de leur enchaînement. Or, le monde du similaire ne peut être qu'un monde marqué. Le visage du monde est couvert de caractères, de chiffres, de mots obscurs et c'est le rôle du poète de les déchiffrer. 
Le grand miroir calme au fond duquel les choses se mirent et se renvoient, l'une l'autre, leurs images, est en réalité tout bruissant de paroles. Les reflets muets sont doublés par des mots qui les indiquent. Connaître, c'est déceler le réseau des ressemblances; être poète, c'est les nommer. Le poète n'est-il pas "celui qui, au-dessous des différences nommées et quotidiennement prévues, retrouve les parentés enfouies des choses, leurs similitudes dispersées". ${ }^{19}$

Pour Agrippa d'Aubigné, il n'y a pas de différence entre les marques visibles que Dieu a déposées sur la surface de la terre pour en faire connaître les secrets intérieurs, et les mots lisibles de l'Écriture, ou les sages de l'Antiquité, qui ont été éclairés par une divine lumière. Le langage n'est pas encore arbitraire, le rapport aux textes est de même nature que le rapport aux choses. Sous sa forme première, quand il fut donné aux hommes par Dieu, le langage était un signe des choses absolument certain et transparent parce qu'il leur ressemblait. Adam, lorsqu'il a imposé leurs premiers noms aux bêtes, n'a fait que lire les marques visibles et silencieuses. Si le langage ne ressemble plus immédiatement aux choses qu'il nomme, il n'est pas pour autant séparé du monde; il continue sous une autre forme, à être le lieu des révélations et à faire partie de l'espace où la vérité, à la fois, se manifeste et s'énonce.

Toute grande poésie est donc mythique, non pas seulement comme oeuvre de l'imagination, mais bien comme annonce de la vérité de l'être qui n'est rendue possible que par l'épiphanie de l'être lui-même. La poésie donne ainsi à voir, en chacune de ses figures, non pas un secteur de l'étant - comme le fait la photographie - mais l'être en son entier. Telle est aussi la raison pour laquelle ses figures sont "dynamiques," tout comme le sont les mythes véritables, c'est-à-dire qu'elles s'emparent de l'âme de telle sorte qu'on lui sait gré de percevoir, fût-ce de façon fugitive, l'être profond de toutes choses et de contempler, comme en un éclair, le visage divin de toute réalité du monde. ${ }^{20}$

La langue poétique n'est nullement un moyen visant à faire comprendre. Dans Les Tragiques, l'oeuvre n'explique pas le combat qui oppose Dieu à Satan; elle ne tente pas d'exprimer son caractère primordial; elle en est la vérité. C'est ce que dit le mot grec mythos: le vrai comme parole. Le divin résonne dans le nom. L'être se donne à reconnaître en des figures sonores. Agrippa d'Aubigné parle parce qu'il lui faut parler, parce que l'être des choses veut devenir manifeste et qu'il trouve à se révéler dans la configuration sonore de la parole.

Plongé dans le chaos de l'histoire, déchiré des pulsions contraires d'une guerre fratricide, d'Aubigné parvient à s'extraire de ce maelstrom et à y instaurer un ordre, tout symbolique qu'il soit, en y inscrivant une césure, le texte. L'acte d'écrire le pose comme sujet de l'énonciation et localise la 
violence en en faisant un signifiant. Dans cet ordre, la souffrance, l'humiliation des Réformés trouve sens. Logos, à nouveau, régne. Or, les rituels religieux - magie, chamanisme, ésotérisme ou autres - le carnaval ou la poésie telle qu'on la pratiquait encore au seizième siècle soulignent les limites du discours social utile et portent témoignage de ce qu'ils refoulent: le procès excédant le sujet et ses structures communicatives, le mythos. A travers la symbolique qu'instaure l'écriture, le langage poétique permet l'émergence de ce qui le travaille, le traverse, le menace; il permet l'ensemble des relations inconscientes, subjectives, sociales dans une attitude d'attaque, d'appropriation, de destruction, de construction, bref, il permet la violence créatrice, l'illumination. Les principes de la ressemblance - métonymie et métaphore - se retrouvent indissociables de l'économie pulsionnelle, alors que la symbolique - syntaxe et toute la catégoralité linguistique - est un produit social du rapport à l'autre. C'est ce rythme sémiotique interne au langage que désigne Mallarmé lorsqu'il parle du "Mystère dans les lettres": indifférent au langage, énigmatique, cet espace sous-jacent à l'écrit est rythmique, déchaîné, irréductible à sa traduction verbale intelligible; il est musical, antérieur au jugé et retenu par une seule garantie, la syntaxe. C'est en reproduisant le signifiant - les guerres de religion - qu'Agrippa d'Aubigné traverse la bordure du symbolique et accède à cette chora sémiotique qui est de l'autre côté de la frontière du social.

"J'ay basti ma Franciade," écrit Ronsard, "sans me soucier si cela est vray ou non". Agrippa d'Aubigné se pose, lui, en porte-parole intransigeant de la Vérité. Les tragiques se soustraient à l'antonomie classique qui opposait histoire et poésie en se soumettant au diktat de la parole vraie, au mythos. L'histoire est alors reconnue comme lieu de révélation et la manifestation de l'esprit se perçoit dans la matière. ${ }^{21}$ On comprend mieux désormais le traitement que fait d'Aubigné de la chronologie. La chronologie, comme telle, appartient au monde du logos. Mais si toute une couche des Tragiques rivalise, par la précision et la qualité de l'information, avec une chronique des guerres de religion, et malgré les intentions avouées d'Agrippa d'Aubigné d'inscrire son oeuvre sous le signe de la raison - nous en voulons pour preuve la forme de cour de justice qu'il lui donne - , sa foi de croyant et sa foi de poète le livrent aux voix du mythos. A travers lui, l'art, cette sémiotisation du symbolique, ré-enchante la vie. Le sacré anime à nouveau le profane; à nouveau profane et sacré ne font qu'un et, dans ce monde réunifié, l'élu réintègre la maison du Père. Le présent, enfin, porte sens. 


\section{Notes}

1. Cf. Frank Lestringant, Agrippa d'Aubigné, les Tragiques (Paris, Presses Universitaires de France, 1986), pp. 7 à 15.

2. Agrippa d'Aubigné, Oeuvres (Paris, Gallimard, "Bibliothèque de la Pléiade," 1969), p. 6.

3. Oeuvres, L. IV, v. 833-4, p. 136.

4. Cf. Mircea Eliade, Aspects du mythe (Paris, Gallimard, 1963), pp. 116-117.

5. Oeuvres, L. I, v. 44, p. 22.

6. Cf. à propos de la valeur du mythe comme modèle exemplaire, Mircea Eliade, Le sacré et le profane (Paris, Gallimard, 1965), pp. 82-86.

7. Oeuvres, L. V, v. 1248, p. 180.

8. Cf. Mircea Eliade, Aspects du mythe, pp. 83-85.

9. Oeuvres, L. III, v. 1059-1062, p. 116.

10. Le christianisme avait condamné le millénarisme comme hérétique. Toutefois, Luther attendait la fin imminente du monde de l'Histoire. Le paradis allait enfin être retrouvé.

11. Oeuvres, L. V, v. 175-6, p. 155.

12. Oeuvres, L. V, v. 179-180, p. 155.

13. Cf. au sujet de la création comme épiphanie du sublime, Walter Friedrich Otto, Dionysos, mythe et culte (Paris, Mercure de France, 1969), 11-51.

14. Cf. Mircea Eliade, Aspects du mythe, p. 149.

15. Oeuvres, L. IV, v. 1389-1392, p. 149.

16. Cf. Walter Friedrich Otto, Dionysos, mythe et culte, pp. 29-31.

17. Oeuvres, L. V, v. 1422-1425, p. 184.

18. Cf. Michel Foucault, Les mots et les choses (Paris, Gallimard, 1966), 7-59.

19. Ibidem, p. 63.

20. Cf. Walter Friedrich Otto, Dionysos, mythe et culte, pp. 99-100. Dionysos lui-même et sa suite faisaient leur entrée dans un vacarme symbolisant le choc spirituel. Il en va de même d'Artemis et de Déméter. Au moment de la plus haute intensité, le vacarme se change en profond silence. Vacarme et silence ne sont que des formes différentes de l'innommé, de l'innommable.

21. Cf. Michel Jeanneret, "Les Tragiques: Mimesis et intertexte," dans Le signe et le texte: étude sur l'écriture au XVI e siècle en France (Lexington, French Forum, 1990), p. 102. 